\title{
Ward Round - A football injury?
}

\section{RT Freeman, WJ Harrison}

1. Cure International Hospital, Blantyre, Malawi

Corresponding author : Robert Freeman, Beit Cure International Hospital, PO Box 31236 Blantyre 3, Malawi. Email: rfreeman@doctors.org.uk $\mathrm{Tel}+2658101031$

\section{Introduction}

A boy aged 12 presented with pain in his left leg and inability to walk. He said that his symptoms started 6 months previously after a fall while playing football. He had increasing pain in his left leg and for the last 3 months he had a discharging sinus from his tibia.

On examination he was apyrexial and appeared poorly nourished. Pulse was regular at 90, BP 110/70 mmHg, with normal heart sounds and the chest was clear. The abdomen was soft and unremarkable. There was skin discolouration of his left tibia, with discharging sinuses and skin loss anteromedially (figure 1). The range of movement of the affected knee was reduced with 5 degrees of fixed flexion and flexion up to 100 degrees.

X-rays are shown across (figure 2)

\section{What is the probable diagnosis?}

How would you manage this?

Continued on page 32
Fig 1 Clinical picture before surgery

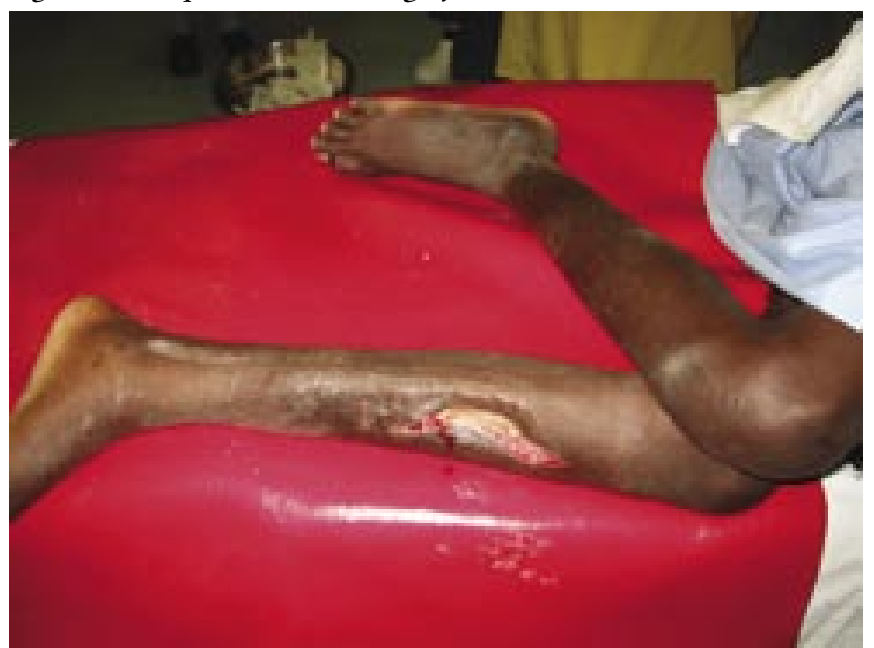

Fig 2 Radiograph of tibia at presentation

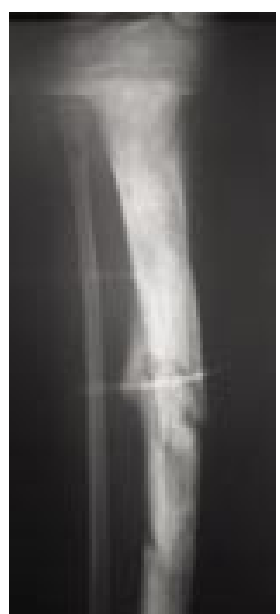

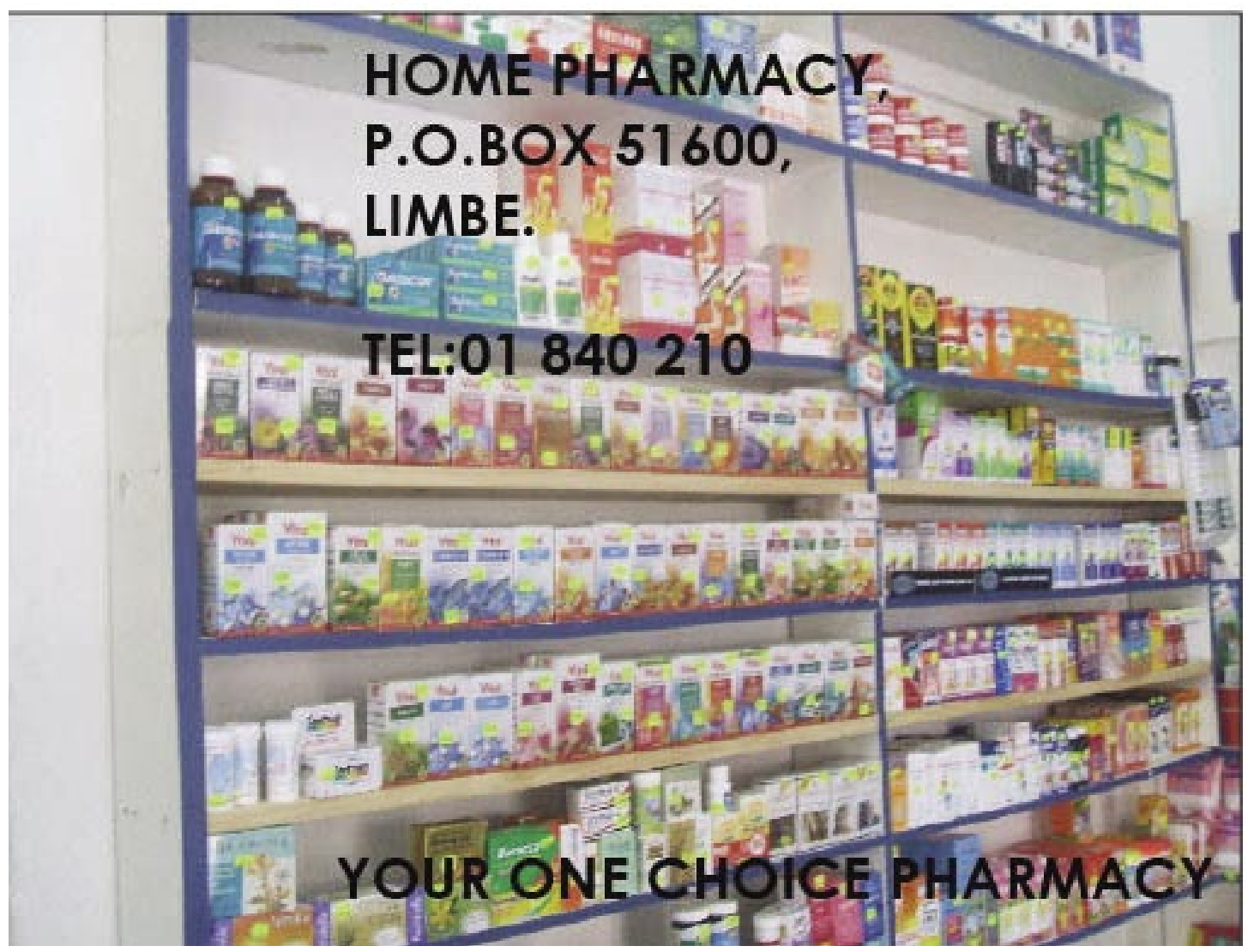

Home Pharmacy is situated at Old Tyresole premises on Milward Road opposite Radio Islam in Limbe. Home Pharmacy has been in operation for five years and specializes in stocking all essential medicines. We keep all branded and generic medicines. Any specialized medicine that is not available in Malawi can be ordered from overseas. 


\section{Ward Round - A football injury? \\ Continued from page 28}

$\mathrm{X}$-rays showed evidence of osteomyelitis involving almost the entire tibial diaphysis, but sparing the proximal and distal growth plates, there was no evidence of involucrum.

In view of the severity of the bony involvement and disability of the child we decided to perform a sequestrectomy even though there was no evidence of involucrum formation.

At surgery the whole tibial diaphysis was found to be dead. A $20 \mathrm{~cm}$ sequestrum was removed leaving the growth plates intact and $1 \mathrm{~cm}$ of healthy bone distally and $3 \mathrm{~cm}$ proximally (figure III). The gap was filled with a block of bone cement containing gentamycin and vancomycin, moulded into the shape of the tibia. The wound edges were approximated to cover the tibial cement spacer, and a plaster applied to immobilise the leg. Post-operatively wounds were dressed daily with honey 1 , oral flucloxacillin was started and the patient was confined to bed.

Three months post sequestrectomy the wounds looked clean. The tibial spacer was removed and an external fixator (Ilizarov frame) was applied to stabilise the tibia and allow further soft tissue healing. At 5 months a non-vascularised free fibula transfer was performed ${ }^{2}$ from the contralateral leg and stabilised with the fixator. A fasciocutaneous flap and split skin graft were performed to improve skin cover.

Two months after the fibula transfer the fine wire fixator was removed. At this stage there were two small sinuses at the proximal end of the graft that were debrided. The post-operative course was complicated by a fracture of the proximal part of the fibula graft. This however healed without intervention. The proximal sinus persisted and required a further debridement and excision of sequestrum. Wounds were left open and treated with daily honey dressings, healing without further problems. Currently the tibia is quiescent, the patient has returned to normal activities, but has been advised not to play contact sports for a further 6 months to allow hypertrophy of the fibula graft (figure $4,5, \& 6$ ).

\section{Discussion}

Patients tend to link the onset of musculoskeletal symptoms with a minor trauma such as a sporting injury. However this is often distracting and a careful history will elicit that the symptoms actually started much earlier. In this case the football injury was almost certainly incidental. Chronic osteomyelitis is a common problem in Malawi. The treatment is surgical excision to eradicate all infected bone. The role of antibiotics is controversial ${ }^{3}$, but antibiotics alone will not eradicate infection as organisms remain in pockets of dead bone (sequestra) with no blood supply and hence antibiotics are unable to reach them. Classical teaching is to allow the involucrum to mature in order to provide structural stability prior to sequestrectomy. However in severe cases involucrum may not form due to thrombosis of both the periosteal and nutrient vessels ${ }^{4}$, and where there is skin breakdown, or the patient is systemically compromised it is inadvisable to wait. If there is no evidence of involucrum formation by 3 months involucrum is unlikely to form and waiting longer is futile ${ }^{1}$.

In these circumstances a sequestrectomy has to be performed
Figure 3 Large sequestrum removed at surgery

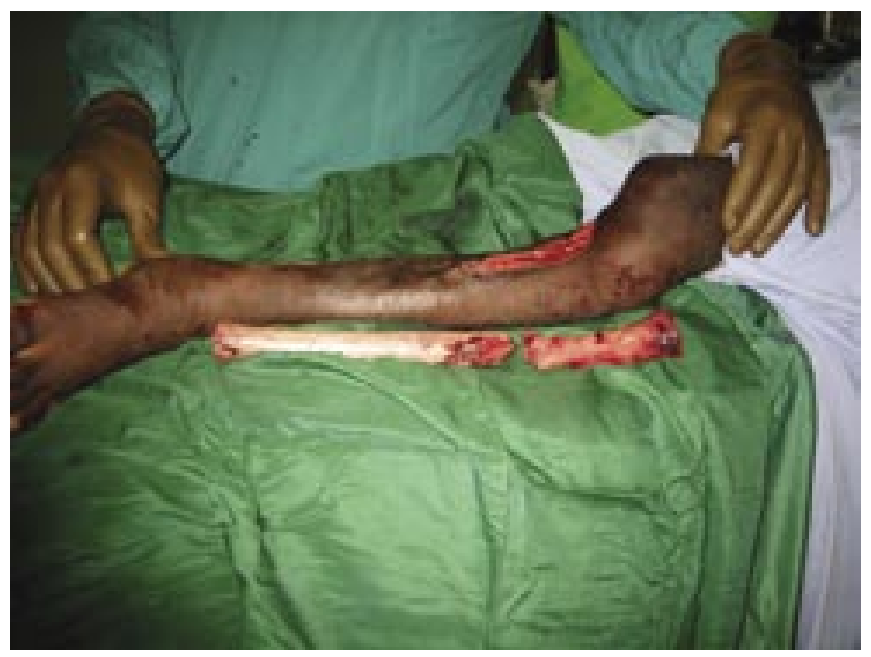

Figure 4 Recent follow up visit appearance

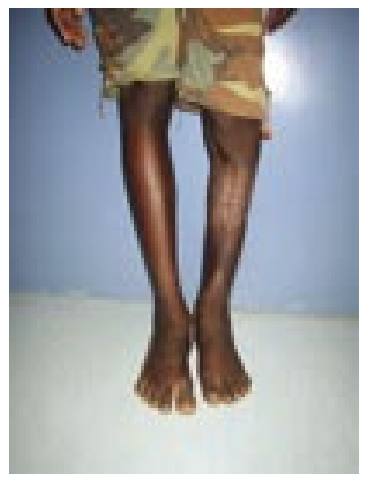

Figure 5 Clinical photograph showing shortening of tibia, but excellent knee flexion

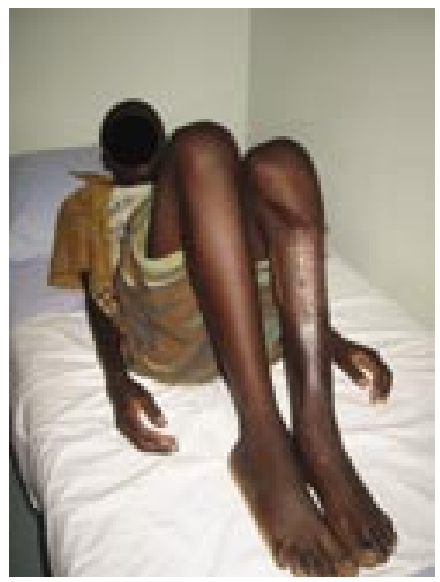

Figure 6 Radiograph of fibula graft incorporated into tibia with evidence of hypertrophy

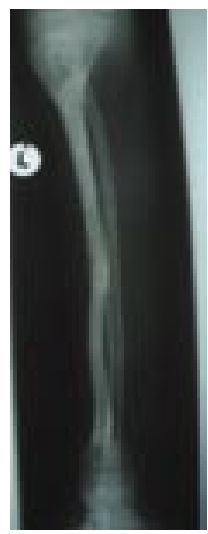


and the defect stabilised by some form of internal or external support. With large defects (especially in children over 6 years old) there is little to be gained by delaying reconstruction, as the gap is likely to be permanent ${ }^{5,6}$. There is much debate over the relative merits of techniques using bone transport ${ }^{7}$, with vascularised ${ }^{8}$ and non-vascularised segments of bone 9. Theoretically a vascularised bone graft brings with it its own blood supply and should therefore help resist infection and allow earlier union. However the technique is time consuming and technically challenging, and in the presence of infection there is a high rate of thrombosis of the vascular pedicle. Non-vascularised grafts are quicker to perform and technically simpler and early reports suggest good results?. Previous reports have described using ipselateral fibula grafts, and stabilising this with a Kirshner wire inserted through the heel. In this case we felt that ipselateral graft harvest would have resulted in further instability of the left leg and elected to take the graft from the contralateral leg. The procedure is not without complication and infection rates of up to $57 \%$ have been reported ${ }^{10}$. However where resources are limited, non-vascularised fibula transfer is a technically simple reliable procedure for filling a large bone defect. Often the only alternative is an above knee amputation. Very long fibula grafts, as in this case, show a high rate of fracture during revascularisation, and take a long time to hypertrophy enough to support the forces a normal tibia withstands. When, as here, a graft fracture heals with callus it is a good indication that revascularisation has occurred.

\section{Conclusion}

Chronic osteomyelitis is a surgical problem which is best treated with sequestrectomy. Generally sequestrectomy is performed when the involucrum has matured to form bony support after removal of the sequestrum. In severe cases involucrum may not form in which case there is often bony discontinuity after the sequestrum has been excised. Nonvascularised fibula transfer is a simple technique for filling and stabilising the resulting defect.

\section{References}

1. Mphande ANG, Killowe C, Phalira S, Wynn Jones H, Harrison WJ. Effects of honey and sugar dressings on wound healing. J Wound Care. 2007 Jul;16 (7):317-9.

2. Morsi E.Tibial reconstruction using a non-vascularised fibular transfer. Int Orthop. 2002;26(6):377-80.

3. Shufford JA, Steckelberg JM Role of oral antimicrobial therapy in the management of osteomyelitis. Curr Opin Infect Dis 2003 Dec;16(6):515-9

4. Daoud A, Saighi-Bouaouina A. Treatment of sequestra, pseudarthrosis and defects in long bones of children who have chronic haematogenous osteomyelitis. J Bone Joint Surg [Am] 1989; 71-A:1448-68.

5. Bosworth DM Subperiosteal resection of the tibial shaft in osteomyelitis. Journal of the American Medical Association 1933; $101,1542$.

6. Griffiths JC, Defects in long bones from severe neglected osteitis J Bone Joint Surg 1968 [Br] 4 813-821.

7. Oh CW, Song HR, Roh JY, Oh JK, Min WK, Kyung HS, Kim JW, Kim PT, Ihn JC. Bone transport over an intramedullary nail for reconstruction of long bone defects in the tibia. Archives of Orthopaedic and Trauma Surgery 2007 1-14

8. Han CS, Wood MB, Bishop AT, Cooney WP Vascularised bone transfer J Bone Joint Surg [Am] 1992 74-A 1441-1449

9. Steinlechner CWB, Mkandawire NC Non-vascularised fibular transfer in the management of defects of long bones after sequestrectomy in children 2005 J Bone Joint Surg [Br] 2005 87-B 1259-63

10. Jupiter JB, Bour CJ, May JW Jr. The reconstruction of defects of the femoral shaft with vascularised transfers of bone. J Bone Joint Surg [Am] 1987; 69-A 365-74.

11.DK Thomas, P Sanchez, EB Hoffman Chronic haematogenous osteomyelitis in children SA Orthopaedic Journal 20076 (4) 8-13 